\title{
Aspirin plus clopidogrel versus aspirin alone for the prevention of coronary artery bypass graft occlusion: angiographic and clinical results of a randomized study
}

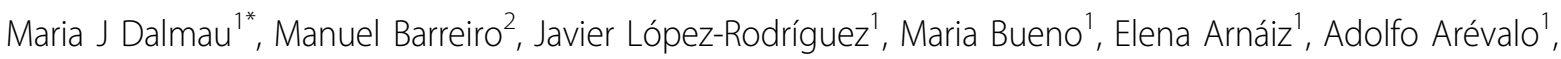 \\ Ana Martín², Jose M González-Santos ${ }^{1}$
}

From World Society of Cardiothoracic Surgeons 25th Anniversary Congress, Edinburgh

Edinburgh, UK. 19-22 September 2015

\section{Background/Introduction}

Prevalence of graft occlusion is high after coronary artery bypass grafting (CABG). Routine use of aspirin after CABG reduces graft failure and ischemic complications. The benefit of concomitant clopidogrel administration remains a controversial issue.

\section{Aims/Objectives \\ We sought to evaluate the impact of use aspirin plus clopi- dogrel versus aspirin alone on graft patency and adverse cardiovascular events 18 months after CABG.}

\section{Method}

In this single-centre prospective randomized study, 200 consecutive patients undergoing elective CABG were randomly assigned to two groups: 97 patients received aspirin $300 \mathrm{mg}$ (A) and 103 patients received aspirin $100 \mathrm{mg}$ plus clopidogrel $75 \mathrm{mg}$ (AC). Antiplatelet protocol was initiated immediately after surgery and continued daily for one year. Graft patency was evaluated by multislice computed tomography angiography (MSCT) 18 months after surgery. The occurrence of major cardiac or cerebrovascular adverse events (MACCE) and bleeding complications during follow-up were assessed.

\section{Results}

Preoperative patient characteristics were similar in both groups. MSCT graft patency was assessed in 194 patients

'Department of Cardiac Surgery, Salamanca University Hospital, Salamanca, 37007, Spain

Full list of author information is available at the end of the article
(97\%). A total of 542 grafts and 680 distal anastomoses were analyzed. Overall graft patency was $83.1 \%$ in group A and $89.4 \%$ in the AC group ( $\mathrm{p}=0.04)$. Patency rates in the A group versus AC group were similar: Left internal mammary artery $(95.9 \%$ vs. $95.1 \%, p=0.85)$, right internal mammary artery $(97.8 \%$ vs. $91.2 \%, \mathrm{p}=0.37)$, radial artery (74.2\% vs. $92.6 \%, \mathrm{p}=0.06)$, saphenous vein graft $(78.6 \%$ vs. $83.5 \%, \mathrm{p}=0.45)$. Clinical follow-up was complete in 200 patients. The incidence of MACCEs was similar in both groups (A 5.2\% vs. AC 9.7\%, p = 0.22). The need for percutaneous coronary re-intervention showed no statistically significant differences (A 3.1\% vs. AC 4.9\%, p = 0.78).

\section{Discussion/Conclusion}

Compared with aspirin monotherapy, the combination of aspirin plus clopidogrel following CABG did not significantly increase graft patency or reduce the incidence of adverse cardiac events.

\section{Authors' details}

'Department of Cardiac Surgery, Salamanca University Hospital, Salamanca, 37007, Spain. ${ }^{2}$ Department of Cardiology, Salamanca University Hospital, Salamanca, 37007, Spain.

Published: 16 December 2015

doi:10.1186/1749-8090-10-S1-A127

Cite this article as: Dalmau et al:: Aspirin plus clopidogrel versus aspirin alone for the prevention of coronary artery bypass graft occlusion: angiographic and clinical results of a randomized study. Journal of Cardiothoracic Surgery 2015 10(Suppl 1):A127. 